\title{
AUTHOR INDEX VOLUME 27 (2015)
}

Abas, W. A. B. W., see Almashaqbeh, S.

Aggarwal, Y., see Chaudhary, N.

Akşahin, M., Erdamar, A., Fırat, H., Ardıç, S. and Eroğul, O., Obstructive Sleep Apnea Classification with Artificial Neural Network Based on Two Synchronic HRV Series

Al-Fahoum, A. and Gharaibeh, K. H., Feasibility Study for ANFIS and EMG

Utilization in Modeling Prosthesis for Trans-Femoral Cut Rehabilitation and Gait Cycle Restoration

Almashaqbeh, S., Al-Sheikh, B., Abas, W. A. B. W. and Osman, N. A. A., Lower Extremities Biomechanics of Regular Stair Climbing: Slim Versus Obese

Al-Sheikh, B., see Almashaqbeh, S.

Amri, H., Hanna, F., Lapayre, J.-C., Khalfallah, A. and Bouhlel, M., Repro: A New Reduction/Expansion Protocol to Increase the Performance of Image Transmission in Medical Telediagnosis Platforms

Ardıç, S., see Akşahin, M.

Arroyo, L., see Guest, B.

Baig, T. N., see Kadir, M. A.

Banerjee, A., Datta, S., Pal, M., Tibarewala, D. N. and Konar, A., Electrooculography

Based Reading Speed Analysis as an Assistive Tool

Behnam, H., see Golchin, E.

Behnam, H., see Shalbaf, R.

Beigzadeh, B., see Halabian, M.

Bijar, A., see Ebrahimzadeh, E.

Blagojević, M., see Topalović, M.

Bouhlel, M., see Amri, H.

Bousbia-Salah, A. and Talha-Kedir, M., Time-Frequency Processing Method of Epileptic EEG Signals

Breloff, S. P. and Chou, L.-S., Influence of Various Daily Tasks on Segmented Trunk Kinematics

Brier, M. E., see Kim, S.

Carreira, J. M., see Suárez-Cuenca, J. J.

Carrero, W., see Cerrolaza, M.

Cedeño, J., see Cerrolaza, M.

Cerrolaza, M., Carrero, W., Cedeño, J. and Valencia, L., Finite Element Analysis of a

Device for Alveolar Osteogenic Distraction in Human Mandible

Cerrolaza, M., see Mangado, N.

Cerrolaza, M., see Uzcátegui, G.

Chan, K.-K., Chen, C.-H., Wu, L.-C., Kuo, Y.-J., Liao, C.-J. and Chiang, C.-J., In

Vivo Evaluation of a New $\beta$-Tricalcium Phosphate Bone Substitute in a Rabbit

Femur Defect Model

Chang, H.-Y., see Yang, S.-C.

Chang, S.-H., see Tzeng, M.-J.
4 (2015) 1550036-000

6 (2015) 1550051-000

2 (2015) 1550011-000

3 (2015) 1550023-000

4 (2015) 1550036-000

4 (2015) 1550036-000

6 (2015) 1550054-000

2 (2015) 1550011-000

5 (2015) 1550045-000

3 (2015) 1550029-000

2 (2015) 1550016-000

6 (2015) 1550052-000

5 (2015) 1550049-000

4 (2015) 1550033-000

4 (2015) 1550038-000

2 (2015) 1550020-000

6 (2015) 1550054-000

2 (2015) 1550015-000

6 (2015) 1550058-000

1 (2015) 1550001-000

2 (2015) 1550012-000

4 (2015) 1550034-000

4 (2015) 1550034-000

4 (2015) 1550034-000

2 (2015) 1550018-000

4 (2015) 1550037-000

3 (2015) 1550028-000

2 (2015) 1550014-000

5 (2015) 1550044-000 
Chaudhary, N., Aggarwal, Y., Singh, N. and Sinha, R. K., Electronic Analogy to Simulate and Predict the Dynamics of Cellular Mechanism of Parkinson's Disease Stimulated by Environmental Factors

Chen, C.-H., see Chan, K.-K.

Chen, C.-Y., see Lee, R.-G.

Chen, P.-C., see Jao, J.-C.

Chen, S.-F., see Yen, J.-H.

Chen, S.-H., see Jao, J.-C.

Chen, S.-H., see $\mathrm{Yu}$, C.

Chen, T., see Lugade, V.

Chern, M.-K., see Yen, J.-H.

Chiang, C.-J., see Chan, K.-K.

Chou, L.-S., see Breloff, S. P.

Chou, L.-S., see Lo, O.-Y.

Chou, L.-S., see Lugade, V.

Chung, P.-C., see Yang, S.-C.

Damnjanović, Đ., see Topalović, M.

Datta, S., see Banerjee, A.

Dávila, E., see Uzcátegui, G.

Du, D., see Zhang, D.

Du, F., see Zhang, D.

Du, H., see Kang, H.

Ebrahimzadeh, E., Pooyan, M., Jahani, S., Bijar, A. and Setaredan, S. K., ECG

Signals Noise Removal: Selection and Optimization of the Best Adaptive Filtering

Algorithm Based on Various Algorithms Comparison

Erdamar, A., see Akşahin, M.

Erickson, C., see Lugade, V.

Eroğul, O., see Akşahin, M.

Farahmand, F., see Hosseininejad, S.

Fateh, H. R., see Hosseininejad, S.

Fatouraee, N., see Rassoli, A.

Feng, Y.-Y., see Hsiao, C.-C.

Filipović, N., see Topalović, M.

Forogh, B., see Hosseininejad, S.

Foroughipour, M., see Zahmati, S.

Firat, H., see Akşahin, M.

Fujimoto, M., see Lugade, V.

Gao, Y., Xu, Y., Luo, Y., Liang, J. and Wang, H., A Breathing Synchronization

Strategy for the Non-Invasive Ventilator System

Gaweda, A. E., see Kim, S.

Gharaibeh, K. H., see Al-Fahoum, A.

Gok, K., Taspinar, F., Inal, S. and Gulbandilar, E., Importance of Sidebar-bone Spacing During the Application of Pertrochanteric Fixator on Femoral Intertrochanteric Fracture Model; Comparison of the Biomechanical Effects Using Finite Element Method

Golchin, E., Setarehdan, S. K. and Behnam, H., Automatic and Concurrent Determination of Optimal Values of Nonlocal Means Filtering Parameters Based on Bayesian Formulation in Ivus Images

Gregersen, H., see Zhang, D.

Guest, B., Arroyo, L., Viel, L., Kerr, C. and Runciman, J., Ex Vivo Equine Heart and Lung Perfusion System
6 (2015) 1550051-000

3 (2015) 1550028-000

6 (2015) 1550055-000

1 (2015) 1550002-000

5 (2015) 1550042-000

1 (2015) 1550002-000

2 (2015) 1550019-000

5 (2015) 1550041-000

5 (2015) $1550042-000$

3 (2015) 1550028-000

6 (2015) 1550058-000

6 (2015) 1550059-000

5 (2015) 1550041-000

2 (2015) 1550014-000

2 (2015) 1550020-000

2 (2015) 1550016-000

4 (2015) 1550037-000

5 (2015) 1550046-000

5 (2015) 1550046-000

1 (2015) 1550008-000

4 (2015) 1550038-000

2 (2015) 1550011-000

5 (2015) 1550041-000

2 (2015) 1550011-000

6 (2015) 1550056-000

6 (2015) 1550056-000

5 (2015) 1550050-000

6 (2015) 1550057-000

2 (2015) 1550020-000

6 (2015) 1550056-000

3 (2015) 1550024-000

2 (2015) 1550011-000

5 (2015) 1550041-000

4 (2015) 1550031-000

1 (2015) 1550001-000

3 (2015) 1550023-000

3 (2015) 1550030-000

6 (2015) 1550052-000

5 (2015) 1550046-000

5 (2015) 1550045-000 
Gulbandilar, E., see Gok, K.

Guler, I., see Ozkaraca, O.

Guo, W., see Suárez-Cuenca, J. J.

Halabian, M., Karimi, A., Beigzadeh, B. and Navidbakhsh, M., A Numerical Study on the Hemodynamic and Shear Stress of Double Aneurysm Through S-Shaped Vessel

Hanna, F., see Amri, H.

Hosseininejad, S., Farahmand, F., Fateh, H. R. and Forogh, B., Subject-Specific and Function-specific Applicability of a Hand-Forearm Musculoskeletal Model

Hou, S.-M., see Shih, K.-S.

Hsiao, C.-C., Lee, R.-G., Tien, S.-C., Feng, Y.-Y. and Huang, S.-F., Early Clinical

Prognosis for High-Risk Chest Pain Patients Using Smart Textiles

Hsiao, C.-C., see Lee, R.-G.

Hsiao, I.-T., see Huang, P.-C.

Hsieh, H.-H., see Huang, P.-C.

Hsieh, M.-S., see Tsai, M.-D.

Hsu, C.-C., see Shih, K.-S.

Hsu, C.-H., see Huang, P.-C.

Hsu, L.-H., see Tzeng, M.-J.

Huang, C.-Y., see Lu, C.-H.

Huang, H.-M., see Lu, C.-H.

Huang, P.-C., Hsieh, H.-H., Hsu, C.-H. and Hsiao, I.-T., An Efficient Sensitivity

Calculation of Tilted Apertures for Preclinical Multi-Pinhole Spect

Huang, S.-F., see Hsiao, C.-C.

Huong, A. K. C. and Ngu, X. T. I., In Situ Monitoring of Mean Blood Oxygen

Saturation Using Extended Modified Lambert Beer Model

Hussain, A., see Saputro, A. H.

Inal, S., see Gok, K.

Jahani, S., see Ebrahimzadeh, E.

Jao, J.-C., Lu, H.-Y., Lu, H.-C., Lian, S.-L., Liu, G.-C., Chen, S.-H., Yang, S.-F. and Chen, P.-C., Monitoring Early Radiation-Induced Liver Injury Using Ferucarbotranenhanced MRI at 3 T: An Animal Model

Jen, C.-C. and Yu, S.-S., Automatic Nipple Detection in Mammograms Using Local Maximum Features Along Breast Contour

Jiang, J., see Pan, M.

Jiang, P., see Lu, L.

Juan, J. G. S., see Lugade, V.

Kadir, M. A., Baig, T. N. and Siddique-e Rabbani, K., Focused Impedance Method to Detect Localized Lung Ventilation Disorders in Combination with Conventional Spirometry

Kang, H., Wang, W., Li, X., Du, H., Li, L. and Ma, J., Multichannel S-EMG System of Masticatory Muscles: Design and Clinical Application in Diagnosis of Dysfunction in Stomatognathic System

Karduna, A., see Lugade, V.

Karimi, A., Kudo, S., Razaghi, R. and Navidbakhsh, M., A Combination of Experimental and Numerical Analyses to Measure the Compressive Mechanical Properties of Tennis Ball

Karimi, A., see Halabian, M.

Kashihara, K., Hybrid Intelligent Controllers for a Multiple Drug Delivery System in Acute Heart Failure

Kerr, C., see Guest, B.

Khalfallah, A., see Amri, H.
3 (2015) 1550030-000

1 (2015) 1550009-000

4 (2015) 1550040-000

4 (2015) 1550033-000

6 (2015) 1550054-000

6 (2015) 1550056-000

6 (2015) 1550060-000

6 (2015) 1550057-000

6 (2015) 1550055-000

1 (2015) 1550006-000

1 (2015) 1550006-000

1 (2015) 1550005-000

6 (2015) 1550060-000

1 (2015) 1550006-000

5 (2015) 1550044-000

1 (2015) 1550007-000

1 (2015) 1550007-000

1 (2015) 1550006-000

6 (2015) 1550057-000

1 (2015) 1550004-000

2 (2015) 1550017-000

3 (2015) 1550030-000

4 (2015) 1550038-000

1 (2015) 1550002-000

4 (2015) 1550035-000

4 (2015) 1550032-000

1 (2015) 1550003-000

5 (2015) 1550041-000

3 (2015) 1550029-000

1 (2015) 1550008-000

5 (2015) 1550041-000

4 (2015) 1550039-000

4 (2015) 1550033-000

5 (2015) 1550043-000

5 (2015) 1550045-000

6 (2015) 1550054-000 
Khalilzadeh, M. M., see Zahmati, S.

3 (2015) 1550024-000

Khang, G., see Lee, C. J.

3 (2015) 1550022-000

Kim, J.-H., see Kim, W.-Y.

Kim, S., Gaweda, A. E., Wu, D., Li, L., Rai, S. N. and Brier, M. E., Simplified

Warfarin Dose-Response Pharmacodynamic Models

Kim, S. Y., see Lee, C. J.

Kim, W.-Y. and Kim, J.-H., Design of Portable EPLDALTERA MAX EPM719SQC160-7 and

3 (2015) 1550025-000

1 (2015) 1550001-000

3 (2015) 1550022-000

DSP $_{\text {TMS320v5410PGE }}$ Application for Non-Invasive In-Extra Cranial Arteries Vascular System

Konar, A., see Banerjee, A.

Koochaki, M., see Niroomand-Oscuii, H.

Kudo, S., see Karimi, A.

Kuo, Y.-J., see Chan, K.-K.

Lan, S.-H., see Yang, S.-C.

Lapayre, J.-C., see Amri, H.

Lee, C. J., Kim, S. Y., Lee, H. G., Yang, J., Park, J. Y., Lee, S. U., Lee, D. and Khang, G., In Vitro Characterization of Tramadol-HCL-loaded Polyoxalate Microspheres Designed for Controlled Drug Release

Lee, D., see Lee, C. J.

Lee, H. G., see Lee, C. J.

Lee, R.-G., Chen, C.-Y., Hsiao, C.-C. and Lin, R., Heart Rate Monitoring Systems in

Groups for Reliability and Validity Assessment of Cardiorespiratory Fitness Analysis

Lee, R.-G., see Hsiao, C.-C.

Lee, S. U., see Lee, C. J.

Li, L., see Kang, H.

Li, L., see Kim, S.

Li, Q., see Suárez-Cuenca, J. J.

Li, X., see Kang, H.

Li, X.-F., see Qiang, N.

Lian, S.-L., see Jao, J.-C.

Liang, H., see Qiang, N.

Liang, J., see Gao, Y.

Liao, C.-J., see Chan, K.-K.

Lin, J.-H., see Lu, C.-H.

Lin, R., see Lee, R.-G.

Liu, G.-C., see Jao, J.-C.

Lo, O.-Y. and Chou, L.-S., Effects of Different Visual Attention Tasks on Obstacle Crossing in Healthy Young Adults

Lozano, L., see Mangado, N.

Lu, C.-H., Lin, J.-H., Huang, H.-M., Huang, C.-Y. and Tai, C.-C., Design of a RingType Reflection Pulse Oximeter with a Parabolic Reflector

Lu, H.-C., see Jao, J.-C.

Lu, H.-Y., see Jao, J.-C.

Lu, L., Yan, G., Zhao, K., Xu, F., Wang, Z. and Jiang, P., Human Gastrointestinal Data Analysis Based on Wavelet Transform-Fractal Algorithm Characterized by

Wireless Capsule

Lu, P.-C., see Yen, J.-H.

Lugade, V., Chen, T., Erickson, C., Fujimoto, M., Juan, J. G. S., Karduna, A. and Chou, L.-S., Comparison of an Electromagnetic and Optical System During Dynamic Motion

Luo, Y., see Gao, Y.

3 (2015) 1550025-000

2 (2015) 1550016-000

3 (2015) 1550026-000

4 (2015) 1550039-000

3 (2015) 1550028-000

2 (2015) 1550014-000

6 (2015) 1550054-000

3 (2015) 1550022-000

3 (2015) 1550022-000

3 (2015) 1550022-000

6 (2015) 1550055-000

6 (2015) 1550057-000

3 (2015) 1550022-000

1 (2015) 1550008-000

1 (2015) 1550001-000

4 (2015) 1550040-000

1 (2015) 1550008-000

6 (2015) 1550053-000

1 (2015) 1550002-000

6 (2015) 1550053-000

4 (2015) 1550031-000

3 (2015) 1550028-000

1 (2015) 1550007-000

6 (2015) 1550055-000

1 (2015) 1550002-000

6 (2015) 1550059-000

2 (2015) 1550018-000

1 (2015) 1550007-000

1 (2015) 1550002-000

1 (2015) 1550002-000

1 (2015) 1550003-000

5 (2015) 1550042-000

5 (2015) 1550041-000

4 (2015) 1550031-000 
Ma, J., see Kang, H.

Mangado, N., Quevedo, C., Lozano, L., Suso, S. and Cerrolaza, M., To What Extent the Combination of Stem Length and Stem Inclination Do Affect the Performance of the Tibial Component in Knee Implants?

Maskon, O., see Saputro, A. H.

Meghdadi, N. and Niroomand-Oscuii, H., Numerical Simulation of the Effect of Venous Needle's Flow Rate and Angle on Flow Parameters of a Hemodialysis Graft

Mohanalakshmi, S. and Sivasubramanian, A., Optical Sensor System for the Non-Invasive Assessment of Arterial Stiffness Quantified by Fourth Derivative of Photoplethysmogram

Mustafa, M. M., see Saputro, A. H.

Nammakie, E., see Niroomand-Oscuii, H.

Navidbakhsh, M., see Halabian, M.

Navidbakhsh, M., see Karimi, A.

Ngu, X. T. I., see Huong, A. K. C.

Niroomand-Oscuii, H., Koochaki, M. and Nammakie, E., An Innovative Method for Generating Pulsatile Blood Flow Via an Axial Ventricular Assist Device

Niroomand-Oscuii, H., see Meghdadi, N.

Nor, I. F. M., see Saputro, A. H.

Osman, N. A. A., see Almashaqbeh, S.

Ozkaraca, O. and Guler, I., Denoising and Remote Monitoring of ECG Signal with Real-Time Extended Kalman Filter in a Wearable System

Pal, M., see Banerjee, A.

Pan, M., Jiang, J., Zhang, F. and Rong, Q., Medical Image Registration Based on Improved Fuzzy C-Means Clustering

Park, J. Y., see Lee, C. J.

Parvez, M. Z. and Paul, M., Novel Approaches of EEG Signal Classification Using IMF Bandwidth and DCT Frequency

Paul, M., see Parvez, M. Z.

Peulić, A., see Topalović, M.

Pooyan, M., see Ebrahimzadeh, E.

Qiang, N., Xie, F., Liang, H., Li, X.-F., Tang, S. and Zhu, J.-X., Synthesis of Poly $[(\varepsilon-\mathrm{Cl})-\mathrm{Co}-(\mathrm{Ga}$-alt- $L$-Asp $)]$ Copolymer and Effects on Cells After Coupling with Small Peptide

Quevedo, C., see Mangado, N.

Rai, S. N., see Kim, S.

Rassoli, A., Fatouraee, N. and Shafigh, M., Uniaxial and Biaxial Mechanical Properties of the Human Saphenous Vein

Razaghi, R., see Karimi, A.

Remy, J., see Suárez-Cuenca, J. J.

Remy-Jardin, M., see Suárez-Cuenca, J. J.

Ren, Q., see Wei, G.

Rong, Q., see Pan, M.

Runciman, J., see Guest, B.

Saini, B. S., see Sethi, G.

Saputro, A. H., Mustafa, M. M., Hussain, A., Zaki, W. M. D. W., Maskon, O. and Nor, I. F. M., Global Feature for Left Ventricular Dysfunction Detection Based on Shape Deformation Tracking

Setaredan, S. K., see Ebrahimzadeh, E.

Setarehdan, S. K., see Golchin, E.
1 (2015) 1550008-000

2 (2015) 1550018-000

2 (2015) 1550017-000

5 (2015) 1550048-000

3 (2015) 1550021-000

2 (2015) 1550017-000

3 (2015) 1550026-000

4 (2015) 1550033-000

4 (2015) 1550039-000

1 (2015) 1550004-000

3 (2015) 1550026-000

5 (2015) 1550048-000

2 (2015) 1550017-000

4 (2015) 1550036-000

1 (2015) 1550009-000

2 (2015) 1550016-000

4 (2015) 1550032-000

3 (2015) 1550022-000

3 (2015) 1550027-000

3 (2015) 1550027-000

2 (2015) 1550020-000

4 (2015) 1550038-000

6 (2015) 1550053-000

2 (2015) 1550018-000

1 (2015) 1550001-000

5 (2015) 1550050-000

4 (2015) 1550039-000

2 (2015) 1550012-000

2 (2015) 1550012-000

1 (2015) 1550010-000

4 (2015) 1550032-000

5 (2015) 1550045-000

5 (2015) 1550047-000

2 (2015) 1550017-000

4 (2015) 1550038-000

6 (2015) 1550052-000 
Sethi, G. and Saini, B. S., Segmentation of Abdomen Diseases Using Active Contour Models in CT Images

Shafigh, M., see Rassoli, A.

Shalbaf, R., Behnam, H. and Sleigh, J., Order Patterns Recurrence Analysis of

Electroencephalogram During Sevoflurane Anesthesia

Shepherd, D. E. T., see Youssef, M. M.

Shih, K.-S., Hsu, C.-C., Zhou, S.-Y. and Hou, S.-M., Biomechanical Investigation of

Pedicle Screw-Based Posterior Stabilization Systems for the Treatment of Lumbar

Degenerative Disc Disease Using Finite Element Analyses

Siddique-e Rabbani, K., see Kadir, M. A.

Singh, N., see Chaudhary, N.

Sinha, R. K., see Chaudhary, N.

Sivasubramanian, A., see Mohanalakshmi, S.

Sleigh, J., see Shalbaf, R.

Souto, M., see Suárez-Cuenca, J. J.

Suárez-Cuenca, J. J., Guo, W. and Li, Q., Integration of Multiple Classifiers for Computerized Detection of Lung Nodules in CT

Suárez-Cuenca, J. J., Souto, M., Tahoces, P. G., Carreira, J. M., Remy-Jardin, M. and

Remy, J., Automatic Detection of Pulmonary Nodules in MDCT: Performance

Evaluation with Independent Datasets

Suso, S., see Mangado, N.

Tahoces, P. G., see Suárez-Cuenca, J. J.

Tai, C.-C., see Lu, C.-H.

Talha-Kedir, M., see Bousbia-Salah, A.

Tan, L., see Zhang, D.

Tang, S., see Qiang, N.

Taspinar, F., see Gok, K.

Tibarewala, D. N., see Banerjee, A.

Tien, S.-C., see Hsiao, C.-C.

Titley, O. G., see Youssef, M. M.

Topalović, M., Damnjanović, Đ., Peulić, A., Blagojević, M. and Filipović, N.,

Syllable-Based Speech Recognition Using Electromyography and Decision Set

Classifier

Tsai, M.-D. and Hsieh, M.-S., A Spine Surgery Simulator Based on Volumetric Models

Tzeng, M.-J., Hsu, L.-H. and Chang, S.-H., Development and Evaluation of a

CAD/3DP Process for Transtibial Socket Fabrication

Uzcátegui, G., Dávila, E. and Cerrolaza, M., A Simple and Efficient Methodology to

Improve Design Proposals of Dental Implants - A Design Case Study

Valencia, L., see Cerrolaza, M.

Viel, L., see Guest, B.

Wang, G., see $\mathrm{Yu}, \mathrm{C}$.

Wang, G., see Zhang, D.

Wang, H., see Gao, Y.

Wang, W., see Kang, H.

Wang, Z., see Lu, L.

Wei, G., Yu, X. and Ren, Q., A Novel Method to Evaluate Human Locomotion Ability

Based on the Finite Element Modeling and Simulation of Musculoskeletal System

$\mathrm{Wu}, \mathrm{D}$. , see Kim, S.

Wu, L.-C., see Chan, K.-K.

Xie, F., see Qiang, N.

$\mathrm{Xu}, \mathrm{F}$., see Lu, L.

5 (2015) 1550047-000

5 (2015) 1550050-000

5 (2015) 1550049-000

2 (2015) 1550013-000

6 (2015) 1550060-000

3 (2015) 1550029-000

6 (2015) 1550051-000

6 (2015) 1550051-000

3 (2015) 1550021-000

5 (2015) 1550049-000

2 (2015) 1550012-000

4 (2015) 1550040-000

2 (2015) 1550012-000

2 (2015) 1550018-000

2 (2015) 1550012-000

1 (2015) 1550007-000

2 (2015) 1550015-000

5 (2015) 1550046-000

6 (2015) 1550053-000

3 (2015) 1550030-000

2 (2015) 1550016-000

6 (2015) 1550057-000

2 (2015) 1550013-000

2 (2015) 1550020-000

1 (2015) 1550005-000

5 (2015) 1550044-000

4 (2015) 1550037-000

4 (2015) 1550034-000

5 (2015) 1550045-000

2 (2015) 1550019-000

5 (2015) 1550046-000

4 (2015) 1550031-000

1 (2015) 1550008-000

1 (2015) 1550003-000

1 (2015) 1550010-000

1 (2015) 1550001-000

3 (2015) 1550028-000

6 (2015) 1550053-000

1 (2015) 1550003-000 
$\mathrm{Xu}, \mathrm{Y} .$, see Gao, Y.

Yan, G., see Lu, L.

Yang, J., see Lee, C. J.

Yang, S.-C., Lan, S.-H., Chang, H.-Y. and Chung, P.-C., Epileptiform Discharges Detection from EEG Signals Using Grouped-Channel Restricted Band Analysis

Yang, S.-F., see Jao, J.-C.

Yen, J.-H., Chen, S.-F., Chern, M.-K. and Lu, P.-C., The Effects of Extensional Stress on Red Blood Cell Hemolysis

Yin, T., see Zhang, D.

Youssef, M. M., Shepherd, D. E. T. and Titley, O. G., Engineering Analysis of a Failed Compass Proximal Interphalangeal (PIP) Joint Hinge

Yu, C., Wang, G. and Chen, S.-H., Numerical Simulation of Flow Field in the Hominine Pharyngeal Cavities of Different Morphology

Yu, S.-S., see Jen, C.-C.

$\mathrm{Yu}, \mathrm{X}$., see Wei, G.

Zahmati, S., Khalilzadeh, M. M. and Foroughipour, M., MRI Edge Detection and Noise Reduction with Hybrid System Based on Wavelet and Curvelet

Zaki, W. M. D. W., see Saputro, A. H.

Zhang, D., Yin, T., Du, F., Du, D., Tan, L., Gregersen, H. and Wang, G., Evaluation of Intimal Hyperplasia and Thrombosis After Implantation of Platelet Glycoprotein IIIa Monoclonal Antibody-Eluting Stent in New Zealand White Rabbit Aorta or Iliac Arteries

Zhang, F., see Pan, M.

Zhao, K., see Lu, L.

Zhou, S.-Y., see Shih, K.-S.

Zhu, J.-X., see Qiang, N.
4 (2015) 1550031-000

1 (2015) 1550003-000

3 (2015) 1550022-000

2 (2015) 1550014-000

1 (2015) 1550002-000

5 (2015) 1550042-000

5 (2015) 1550046-000

2 (2015) 1550013-000

2 (2015) 1550019-000

4 (2015) 1550035-000

1 (2015) 1550010-000

3 (2015) 1550024-000

2 (2015) 1550017-000

5 (2015) 1550046-000

4 (2015) 1550032-000

1 (2015) 1550003-000

6 (2015) 1550060-000

6 (2015) 1550053-000 